\title{
Some Almost Sure Convergence Theorems for Branching Processes
}

\author{
C. C. HEYDE
}

\section{Introduction}

In this paper, we shall be concerned with a super-critical Galton-Watson process $\left\{Z_{0}=1, Z_{1}, Z_{2}, \ldots\right\}$ whose non-degenerate offspring distribution has $1<m=E Z_{1}<\infty$ and var $Z_{1}=\sigma^{2}<\infty$. The Galton-Watson process evolves in such a way that the probability generating function of $Z_{n}$ is the $n$-th functional iterate of the probability generating function of $Z_{1}$.

It is well-known that, for the process in question, $m^{-n} Z_{n}$ converges almost surely (a.s.) to a non-degenerate random variable $W$ as $n \rightarrow \infty$ (e.g. Harris [2], p. 13). Also, a central limit analogue holds, namely that, conditional on $Z_{n}>0$, $\left(m^{2}-m\right)^{\frac{1}{2}} \sigma^{-1} Z_{n}^{-\frac{1}{2}}\left(m^{n} W-Z_{n}\right)$ converges in distribution to the unit normal (Heyde [3]). It is an important question as to whether there is, in addition, an iterated logarithm analogue and it is the object of this paper to show that this is the case. Corresponding results also hold for continuous time Markov branching processes and can be obtained by minor modifications of the present arguments. In this connection, we mention that an iterated logarithm analogue for the pure birth process has been obtained by Kendall [5] but his methods are quite different. We make extensive use of results on rates of convergence to normality which were established in [4].

\section{Results}

Theorem. Suppose that $E Z_{1}^{3}<\infty$. Then, on the non-extinction set $\{W>0\}$ we have almost surely,

and

$$
\limsup _{n \rightarrow \infty} \frac{Z_{n+r}-m^{r} Z_{n}}{\left(2 \sigma_{r}^{2} Z_{n} \log n\right)^{\frac{1}{2}}}=1, \quad \liminf _{n \rightarrow \infty} \frac{Z_{n+r}-m^{r} Z_{n}}{\left(2 \sigma_{r}^{2} Z_{n} \log n\right)^{\frac{1}{2}}}=-1
$$

$$
\begin{aligned}
& \limsup _{n \rightarrow \infty} \frac{m^{n} W-Z_{n}}{\left(2 \sigma^{2}\left(m^{2}-m\right)^{-1} Z_{n} \log n\right)^{\frac{1}{2}}}=1, \\
& \liminf _{n \rightarrow \infty} \frac{m^{n} W-Z_{n}}{\left(2 \sigma^{2}\left(m^{2}-m\right)^{-1} Z_{n} \log n\right)^{\frac{1}{2}}}=-1,
\end{aligned}
$$

where $r \geqq 1$ is any fixed integer and

$$
\sigma_{r}^{2}=\operatorname{var} Z_{r}=\sigma^{2} m^{r}\left(m^{r}-1\right)\left(m^{2}-m\right)^{-1} .
$$

Proof. We shall just obtain the results for lim sup; those for lim inf require only minor modifications to the arguments given. There is also no essential loss of generality in giving the proof for the case $P\left(Z_{1}=0\right)=0$ in which case $Z_{n} \rightarrow \infty$ 
with probability one as $n \rightarrow \infty$. We shall do this for the sake of convenience. When $P\left(Z_{1}=0\right)>0$, it is well known that $Z_{n} \rightarrow \infty$ a. s. on the set $\{W>0\}$.

Firstly we shall work on (1). We have, using Theorem 2 of [4], that

$$
\sum_{n=1}^{\infty} \sup _{x}\left|P\left(\sigma_{r}^{-1} Z_{n}^{-\frac{1}{2}}\left(Z_{r+n}-m^{r} Z_{n}\right) \leqq x\right)-\Phi(x)\right|<\infty,
$$

$\Phi(x)$ being the distribution function of the unit normal. Thus, for any $\delta>0$,

$$
\sum_{n=2}^{\infty} P\left(\sigma_{r}^{-1} Z_{n}^{-\frac{1}{2}}\left(Z_{r+n}-m^{r} Z_{n}\right)>(1+\delta)(2 \log n)^{\frac{1}{2}}\right)<\infty,
$$

since $\sum_{n=2}^{\infty}\left[1-\Phi\left((1+\delta)(2 \log n)^{\frac{1}{2}}\right)\right]<\infty$ and hence, from the Borel-Cantelli lemma,

$$
\limsup _{n \rightarrow \infty} \frac{Z_{r+n}-m^{r} Z_{n}}{\left(2 \sigma_{r}^{2} Z_{n} \log n\right)^{\frac{1}{2}}} \leqq 1
$$

with probability one.

Next, we shall fix attention on the particular case $r=1$. For $0<\delta<1$, define

$$
A_{n}=\left\{Z_{n}-m Z_{n-1}>(1-\delta) \sigma\left(2 Z_{n-1} \log (n-1)\right)^{\frac{1}{2}}\right\},
$$

$n=2,3, \ldots$ and note that $A_{n} \in \mathscr{F}\left(Z_{1}, \ldots, Z_{n}\right)$, the $\sigma$-field generated by $Z_{1}, \ldots, Z_{n}$. We make use of an extension of the Borel-Cantelli lemma which gives $P\left(A_{n}\right.$ i. o. $)=1$ if and only if $\sum_{n=1}^{\infty} P\left(A_{n+1} \mid Z_{n}, \ldots, Z_{1}\right)=\infty$ with probability one (e.g. Breiman [1], Corollary 5.29, p. 96).

Now,

$$
\begin{aligned}
& P\left(A_{n+1} \mid Z_{n}, \ldots, Z_{1}\right) \\
& \quad=P\left(Z_{n+1}-m Z_{n}>(1-\delta) \sigma\left(2 Z_{n} \log n\right)^{\frac{1}{2}} \mid Z_{n}, \ldots, Z_{1}\right) \\
& \quad=P\left(\left(Z_{1}^{(1)}-m\right)+\cdots+\left(Z_{1}^{\left(Z_{n}\right)}-m\right)>(1-\delta) \sigma\left(2 Z_{n} \log n\right)^{\frac{1}{2}} \mid Z_{n}\right),
\end{aligned}
$$

where the $Z_{1}^{(i)}$ are independent and identically distributed each with the distribution of $Z_{1}$ and are independent of $Z_{1}, \ldots, Z_{n}$. Thus, using the Berry-Esseen inequality,

$$
\begin{aligned}
& P\left(\left(Z_{1}^{(1)}-m\right)+\cdots+\left(Z_{1}^{\left(Z_{n}\right)}-m\right)>(1-\delta) \sigma\left(2 Z_{n} \log n\right)^{\frac{1}{2}} \mid Z_{n}\right) \\
& \quad \geqq 1-\Phi\left((1-\delta)(2 \log n)^{\frac{1}{2}}\right)-c Z_{n}^{-\frac{1}{2}}
\end{aligned}
$$

$c$ being a positive constant. Also,

since

$$
\sum_{n=2}^{\infty}\left[1-\Phi\left((1-\delta)(2 \log n)^{\frac{1}{2}}\right)\right]=\infty \quad \text { and } \quad \sum_{n=1}^{\infty} Z_{n}^{-\frac{1}{2}}<\infty \quad \text { a.s. }
$$

using Theorem 2 of [4]. Thus,

$$
\sum_{n=1}^{\infty} E\left(Z_{n}^{-\frac{1}{2}}\right) \leqq \sum_{n=1}^{\infty}\left(E Z_{1}^{-1}\right)^{+n / 2}<\infty
$$

$$
\sum_{n=1}^{\infty} P\left(A_{n+1} \mid Z_{n}, \ldots, Z_{1}\right)=\infty
$$


with probability one, from which we deduce that $P\left(A_{n}\right.$ i. o. $)=1$. This gives

$$
\limsup _{n \rightarrow \infty} \frac{Z_{n+1}-m Z_{n}}{\left(2 \sigma^{2} Z_{n} \log n\right)^{\frac{1}{2}}} \geqq 1
$$

with probability one and the particular case $r=1$ of the lim sup part of (1) follows from (4) and (5).

To deal with the case $r>1$, we write $Z_{n}^{*}$ for $Z_{r n}, n=0,1,2, \ldots$ The process $\left\{Z_{n}^{*}\right\}$ is a super-critical Galton-Watson process with offspring distribution that of $Z_{r}$. Thus, applying the lim sup part of (1) with $r=1$ to the process $\left\{Z_{n}^{*}\right\}$, we have with probability one,

which gives

$$
\limsup _{n \rightarrow \infty} \frac{Z_{n+1}^{*}-m^{r} Z_{n}^{*}}{\left(2 \sigma_{r}^{2} Z_{n}^{*} \log n\right)^{\frac{1}{2}}}=1
$$

$$
\limsup _{n \rightarrow \infty} \frac{Z_{n r+r}-m^{r} Z_{n r}}{\left(2 \sigma_{r}^{2} Z_{n r} \log n r\right)^{\frac{1}{2}}}=1
$$

with probability one. But,

$$
\limsup _{n \rightarrow \infty} \frac{Z_{n+r}-m^{r} Z_{n}}{\left(2 \sigma_{r}^{2} Z_{n} \log n\right)^{\frac{1}{2}}} \geqq \limsup _{n \rightarrow \infty} \frac{Z_{n r+r}-m^{r} Z_{n r}}{\left(2 \sigma_{r}^{2} Z_{n r} \log n r\right)^{\frac{1}{2}}},
$$

which together with (4) gives the lim sup part of (1). The lim inf part follows similarly.

In order to obtain (2) we firstly use Theorem 2 of [4] to deduce that

$$
\sum_{n=1}^{\infty} \sup _{x}\left|P\left(\left(m^{2}-m\right)^{\frac{1}{2}} \sigma^{-1} Z_{n}^{-\frac{1}{2}}\left(Z_{n}-m^{n} W\right) \leqq x\right)-\Phi(x)\right|<\infty,
$$

from which we obtain, for any $\delta>0$,

$$
\sum_{n=2}^{\infty} P\left(\left|Z_{n}-m^{n} W\right|>(1+\delta)\left(2 \sigma^{2}\left(m^{2}-m\right)^{-1} Z_{n} \log n\right)^{\frac{1}{2}}\right)<\infty
$$

since $\sum_{n=2}^{\infty}\left[1-\Phi\left((1+\delta)(2 \log n)^{\frac{1}{2}}\right)+\Phi\left(-(1+\delta)(2 \log n)^{\frac{1}{2}}\right)\right]<\infty$. Thus, from the Borel-Cantelli lemma,

with probability one.

$$
\limsup _{n \rightarrow \infty} \frac{\left|Z_{n}-m^{n} W\right|}{\left(2 \sigma^{2}\left(m^{2}-m\right)^{-1} Z_{n} \log n\right)^{\frac{1}{2}}} \leqq 1
$$

Next, making use of (1) we have with probability one and for any fixed integer $r \geqq 1$,

so that

$$
1=\limsup _{n \rightarrow \infty} \frac{Z_{r+n}-m^{r} Z_{n}}{\left(2 \sigma^{2} m^{r}\left(m^{r}-1\right)\left(m^{2}-m\right)^{-1} Z_{n} \log n\right)^{\frac{1}{2}}},
$$

$$
\begin{aligned}
& \left(1-\frac{1}{m^{r}}\right)^{\frac{1}{2}}=\limsup _{n \rightarrow \infty} \frac{\left(m^{-r} Z_{r+n}-W m^{n}\right)+\left(W m^{n}-Z_{n}\right)}{\left(2 \sigma^{2}\left(m^{2}-m\right)^{-1} Z_{n} \log n\right)^{\frac{1}{2}}} \\
& \quad \leqq \limsup _{n \rightarrow \infty} \frac{m^{-r} Z_{r+n}-W m^{n}}{\left(2 \sigma^{2}\left(m^{2}-m\right)^{-1} Z_{n} \log n\right)^{\frac{1}{2}}}+\limsup _{n \rightarrow \infty} \frac{W m^{n}-Z_{n}}{\left(2 \sigma^{2}\left(m^{2}-m\right)^{-1} Z_{n} \log n\right)^{\frac{1}{2}}}
\end{aligned}
$$


with probability one. But,

$$
\begin{aligned}
& \limsup _{n \rightarrow \infty} \frac{m^{-r} Z_{r+n}-W m^{n}}{\left(2 \sigma^{2}\left(m^{2}-m\right)^{-1} Z_{n} \log n\right)^{\frac{1}{2}}} \\
& \quad=\frac{1}{m^{r / 2}} \limsup _{n \rightarrow \infty} \frac{Z_{r+n}-W m^{r+n}}{\left(2 \sigma^{2}\left(m^{2}-m\right)^{-1} Z_{r+n} \log (r+n)\right)^{\frac{1}{2}}}\left(\frac{Z_{r+n} \log (r+n)}{m^{r} Z_{n} \log n}\right)^{\frac{1}{2}} \leqq \frac{1}{m^{r / 2}}
\end{aligned}
$$

with probability one in view of (7) and since $m^{r} Z_{n}^{-1} Z_{r+n} \stackrel{\text { a.s. }}{\longrightarrow} 1$ as $n \rightarrow \infty$. Therefore, using (9) in (8), we have with probability one that

$$
\limsup _{n \rightarrow \infty} \frac{W m^{n}-Z_{n}}{\left(2 \sigma^{2}\left(m^{2}-m\right)^{-1} Z_{n} \log n\right)^{\frac{1}{2}}} \geqq\left(1-\frac{1}{m^{r}}\right)^{\frac{1}{2}}-\frac{1}{m^{r / 2}} .
$$

Now, (10) holds for any integer $r \geqq 1$ so we must have

$$
\limsup _{n \rightarrow \infty} \frac{W m^{n}-Z_{n}}{\left(2 \sigma^{2}\left(m^{2}-m\right)^{-1} Z_{n} \log n\right)^{\frac{1}{2}}} \geqq 1
$$

with probability one. (7) and (11) then give the required lim sup part of (2). Similar reasoning gives the lim inf part.

\section{Concluding Remark}

The condition $E Z_{1}^{3}<\infty$ in the theorem can at least be weakened to $E Z_{1}^{2+\delta}<\infty$, some $\delta>0$. This is a simple consequence of the fact that (3) and (6) and a minor modification of the Berry-Esseen bound continue to hold under the condition $E Z_{1}^{2+\delta}<\infty$. See the remarks after the proof of Theorem 2 of [4] concerning geometric bounds on the rate of convergence to normality for the case $E Z_{1}^{2+\delta}<\infty$.

Note Added in Proof. Subsequent research has revealed that the theorem of this paper continues to hold under the condition $E Z_{1}^{2}<\infty$. This result will appear in a paper of Heyde and Leslie in Bull. Austral. math. Soc. 5 (1971).

\section{References}

1. Breiman, L.: Probability. Reading, Mass.: Addison-Wesley 1968.

2. Harris, T.E.: The theory of branching processes. Berlin-Göttingen-Heidelberg: Springer 1963.

3. Heyde, C.C.: Some central limit analogues for super-critical Galton-Watson processes. J. appl. Probab. 8, 52-59 (1971).

4. - Brown, B. M.: An invariance principle and some convergence rate results for branching processes. Z. Wahrscheinlichkeitstheorie verw. Geb. (1971) (to appear).

5. Kendall, D.G.: Branching processes since 1873. J. London math. Soc. 41, 385-406 (1966).

Dr. C.C. Heyde

Department of Statistics

Australian National University

School of General Studies

Box 4, P.O. Canberra, A.C.T. 2600

Australia 